\title{
A Reverse-Shock Model for the Early Afterglow of GRB 050525A
}

\author{
L. Shao and Z. G. Dai \\ Department of Astronomy, Nanjing University, Nanjing 210093, China; dzg@nju.edu.cn
}

\begin{abstract}
The prompt localization of gamma-ray burst (GRB) 050525A by Swift allowed the rapid follow-up of the afterglow. The observations revealed that the optical afterglow had a major rebrightening starting at $\sim 0.01$ days and ending at $\sim 0.03$ days, which was followed by an initial power-law decay. Here we show that this early emission feature can be interpreted as the reverse shock emission superposed by the forward shock emission in an interstellar medium environment. By fitting the observed data, we further constrain some parameters of the standard fireballshock model: the initial Lorentz factor of the ejecta $\gamma_{0}>120$, the magnetic energy fraction $\epsilon_{B}>4 \times 10^{-6}$, and the medium density $n<2 \mathrm{~cm}^{-3}$. These limits are consistent with those from the other very-early optical afterglows observed so far. In principle, a wind environment for GRB 050525A is disfavored.
\end{abstract}

Subject headings: gamma rays: bursts — relativity — shock waves

\section{Introduction}

The gamma-ray burst (GRB) 050525A is a bright brief flash of gamma-ray radiation detected by the Swift Burst Alert Telescope (BAT) on 25 May 2005 00:02:53 (UT) (Band et al. 2005). It showed two peaks, with a duration of $T_{90}=8.8 \pm 0.5 \mathrm{~s}$ in the $15-350 \mathrm{keV}$ band (Markwardt et al. 2005; Cummings et al. 2005). Fitting to the Band spectral model yields a low-energy photon index of $\alpha=1.0 \pm 0.1$ and a peak energy of $E_{p}=79 \pm 4 \mathrm{keV}$ (Cummings et al. 2005). The fluence in the $15-350 \mathrm{keV}$ band is $(2.0 \pm 0.1) \times 10^{-5} \mathrm{ergs} \mathrm{cm}^{-2}$. This burst

was also detected by other on-board instruments such as INTEGRAL and Konus-Wind, with peak fluxes of $\sim 3.2 \times 10^{-6} \mathrm{ergs} \mathrm{cm}^{-2} \mathrm{~s}^{-1}$ (Gotz et al. 2005) and $\sim 8.7 \times 10^{-6} \mathrm{ergs} \mathrm{cm}^{-2} \mathrm{~s}^{-1}$ (Golenetskii et al. 2005), respectively. The fluences were $\geq 1.2 \times 10^{-5} \mathrm{ergs} \mathrm{cm}^{-2}$ in $20-200$ $\mathrm{keV}$ (for the 12 second integration time) by INTEGRAL and $\sim 7.8 \times 10^{-5} \mathrm{ergs} \mathrm{cm}^{-2}$ by Konus-Wind in 20-1000 keV (for the 11.5 second integration time), respectively. The timeintegrated spectrum shows a peak energy $E_{p}=84.1 \pm 1.7 \mathrm{keV}$ by Konus-Wind (Golenetskii et al. 2005). About six minutes later, the ROTSE III telescope in Namibia was able to 
obtain images, and detected an optical afterglow of magnitude 14.7 (Rykoff, Yost, \& Swan 2005). A presumed host galaxy was measured with a redshift of $\mathrm{z}=0.606$, based on both [O III] 5007 and $H_{\beta}$ emission and Ca H\&K and Ca I 4228 absorption (Foley et al. 2005). Assuming cosmological parameters $\Omega_{M}=0.27, \Omega_{\Lambda}=0.73$, and $H_{0}=71 \mathrm{~km} \mathrm{~s}^{-1} \mathrm{Mpc}^{-1}$, the isotropic-equivalent gamma-ray energy is about $1.2 \times 10^{53}$ ergs.

The follow-up observations of GRB 050525A revealed that the optical afterglow decayed as $\propto t^{-1.3}$ until about 0.01 days after the burst. Subsequently the afterglow showed a major rebrightening starting at $\sim 0.01$ days and ending at $\sim 0.03$ days, and then decayed as $\propto t^{-1.0}$. This early-time emission feature is similar to the behavior of external reverse shock emission predicted by the standard fireball-shock model (Mészáros \& Rees 1997, 1999; Sari \& Piran 1999a), which was first confirmed by the detections of early prompt optical emission from GRB 990123 (Akerlof et al. 1999; Sari \& Piran 1999b). The reverse shock model was also suggested to interpret early optical emission of GRB 021004 (Fox et al. 2003a; Kobayashi \& Zhang 2003a) and GRB 021211 (Fox et al. 2003b; Li et al. 2003; Wei 2003; Panaitescu \& Kumar 2004b). Besides, the prompt optical-infrared emission from GRB 041219A was recently suggested to be the internal shock emission, since a strong correlation between $\gamma$ ray and optical-infrared signals was detected (Vestrand et al. 2005; Blake et al. 2005), which further confirmed the standard internal-external-shock model of GRBs. Mirabal et al. (2005) reported a break at $\sim 0.4$ days in the optical afterglow light curve of GRB $050525 \mathrm{~A}$. In this paper, we show that the optical afterglow in $\sim 0.4$ days after the Swift trigger can be well understood due to the reverse shock emission superposed by the forward shock emission.

\section{Optical Emission from Forward and Reverse Shocks}

The standard model for GRBs and their afterglows is the fireball-shock model (for recent review articles, see Mészáros 2002; Zhang \& Mészáros 2004; Piran 2005). In this model, the prompt emission of GRBs is ascribed to internal shocks and the long-term afterglow to external shocks. The prompt or very early optical emission is therefore explained as a consequence of the reverse component of the external shocks (Mészáros \& Rees 1997; Sari \& Piran 1999b).

For shock-accelerated slow-cooling electrons with a power-law distribution, the typical spectrum of synchrotron emission is described by three power laws (Sari, Piran, \& Narayan 1998): (1) $F_{\nu}=\left(\nu / \nu_{m}\right)^{1 / 3} F_{\nu, \max }$ for $\nu<\nu_{m}$; (2) $F_{\nu}=\left(\nu / \nu_{m}\right)^{-(p-1) / 2} F_{\nu, \max }$ for $\nu_{m}<\nu<\nu_{c}$; (3) $F_{\nu}=\left(\nu_{c} / \nu_{m}\right)^{-(p-1) / 2}\left(\nu / \nu_{c}\right)^{-p / 2} F_{\nu, \max }$ for $\nu>\nu_{c}$, where $\nu_{m}, \nu_{c}$ and $F_{\nu, \text { max }}$ are the typical synchrotron frequency, cooling frequency and peak flux, respectively. The parameter $p$ is the index of the electron energy distribution. Here we ignore the synchrotron self-absorption 
because its corresponding frequency may be much less than the optical band of interest.

Both a forward shock and a reverse shock emerge when an ultrarelativistic cold GRB ejecta with initial Lorentz factor of $\gamma_{0}$ sweeps up a stationary cold interstellar medium: the forward shock propagates into the interstellar medium and the reverse shock propagates back into the ejecta (Katz 1994; Sari \& Piran 1995). The emission of the forward shock is characterized by (Sari, Piran, \& Narayan 1998)

$$
\begin{aligned}
\nu_{m, f}= & 4.6 \times 10^{11}\left(\frac{1+z}{2}\right)^{1 / 2}\left(\frac{E_{\text {iso }}}{10^{53} \mathrm{ergs}}\right)^{1 / 2}\left(\frac{\epsilon_{B}}{10^{-3}}\right)^{1 / 2}\left(\frac{\epsilon_{e}}{10^{-1}}\right)^{2}\left(\frac{g_{m}}{0.087}\right) t_{d}^{-3 / 2} \mathrm{~Hz},(1) \\
\nu_{c, f}= & 5.8 \times 10^{16}\left(\frac{1+z}{2}\right)^{-1 / 2}\left(\frac{E_{\text {iso }}}{10^{53} \mathrm{ergs}}\right)^{-1 / 2}\left(\frac{\epsilon_{B}}{10^{-3}}\right)^{-3 / 2}\left(\frac{g_{c}}{0.128}\right) n^{-1} t_{d}^{-1 / 2} \mathrm{~Hz}, \\
t_{m, f}= & 1.1 \times 10^{-2}\left(\frac{1+z}{2}\right)^{1 / 3}\left(\frac{E_{\text {iso }}}{10^{53} \mathrm{ergs}}\right)^{1 / 3}\left(\frac{\epsilon_{B}}{10^{-3}}\right)^{1 / 3}\left(\frac{\epsilon_{e}}{10^{-1}}\right)^{4 / 3}\left(\frac{g_{m}}{0.087}\right)^{2 / 3} \\
& \times\left(\frac{\nu_{R}}{10^{15} \mathrm{~Hz}}\right)^{-2 / 3} \text { days, } \\
F_{\nu, \text { max }, f}= & 25\left(\frac{1+z}{2}\right)\left(\frac{E_{\text {iso }}}{10^{53} \mathrm{ergs}}\right)\left(\frac{\epsilon_{B}}{10^{-3}}\right)^{1 / 2}\left(\frac{D_{L}}{10^{28} \mathrm{~cm}}\right)^{-2}\left(\frac{g_{\text {max }}}{2.44}\right) n^{1 / 2} \mathrm{mJy},
\end{aligned}
$$

where, $t_{m, f}$ is the critical times when the break frequency, $\nu_{m, f}$ crosses the observed frequency $\nu_{R}, \epsilon_{B}$ is the fraction of the shock energy goes into the magnetic field, $\epsilon_{e}$ is the fraction of the shock energy goes into the electrons, $g_{m}=(p-0.67)(p-2)^{2} /(p-1)^{2}, g_{c}=(p-0.46) e^{-1.16 p}$, $g_{\max }=p+0.14$ (Granot \& Sari 2002), $E_{\text {iso }}$ is the isotropic-equivalent kinetic energy, $n$ is the density of interstellar medium in units of $1 \mathrm{~cm}^{-3}, z$ is the redshift of the burst, $D_{L}$ is the corresponding luminosity distance, and $t_{d}$ is the observer's time in units of 1 day. The above equations are valid for $p>2$. Afterglows with the hard electron spectrum of $1<p<2$ have been discussed by Dai \& Cheng (2001).

For reverse shocks, it is possible to get a simple analytic solution in two limiting cases: thin shell and thick shell (which are corresponding to Newtonian and relativistic reverse shock, respectively; Sari \& Piran, 1995). Since the reverse-shocked gas is separated with the forward-shocked gas by a contact discontinuity, which keeps the equality of pressures and velocities in both shocks, we can find the emission properties of reverse shock in the aid of the correlations between forward and reverse shocks at the crossing time $t_{\times}$(Kobayashi 2000; Kobayashi \& Zhang 2003a). First, since the reverse- and forward-shocked gases have the same magnetic field and Lorentz factor at $t_{\times}$, the cooling frequency of the reverse-shocked 
electrons is equal to that of the forward-shocked electrons, $\nu_{c, r}\left(t_{\times}\right)=\nu_{c, f}\left(t_{\times}\right)$. Second, the electron's random Lorentz factor of the reverse shock is smaller than that of the forward shock by a factor of $\gamma_{0} / \gamma_{\times}^{2}$ (where $\gamma_{\times}$is the Lorentz factor of both shocked regions at $t_{\times}$), the typical synchrotron frequency of the reverse shock is by a factor of $\gamma_{0}^{2} / \gamma_{x}^{4}$ less than that of the forward shock, $\nu_{m, r}\left(t_{\times}\right)=\left(\gamma_{0}^{2} / \gamma_{\times}^{4}\right) \nu_{m, f}\left(t_{\times}\right)$. This is basically valid for the thick shell case. As for a thin shell, it is more realistic with the expression, $\nu_{m, r}\left(t_{\times}\right)=\left(\gamma_{34, \times}-1\right)^{2} /\left(\gamma_{\times}-1\right)^{2} \nu_{m, f}\left(t_{\times}\right)$, where $\gamma_{34, \times} \simeq\left(\gamma_{\times} / \gamma_{0}+\gamma_{0} / \gamma_{\times}\right) / 2$ is the Lorentz factor of the shocked ejecta in the rest frame comoving with the unshocked ejecta at the crossing time and $\gamma_{x} \simeq \gamma_{0} / 2$ (Zhang, Kobayashi, \& Mészáros 2003; Fan \& Wei 2005). Third, the peak flux $F_{\nu, \max }$ of an emission region is proportional to the electron number, the magnetic field, and the Lorentz boost. Since at $t_{\times}$, the electron number of the ejected shell is larger than that of the swept-up medium by a factor of $\gamma_{\times}^{2} / \gamma_{0}$, we obtain $F_{\nu, \max , r}\left(t_{\times}\right)=\left(\gamma_{\times}^{2} / \gamma_{0}\right) F_{\nu, \max , f}\left(t_{\times}\right)$. Therefore, we can directly write down the corresponding characteristic variables of the reverse shock emission, at $t=t_{\times}$,

$$
\begin{aligned}
\nu_{m, r}\left(t_{\times}\right)= & 4.6 \times 10^{11}\left(\frac{1+z}{2}\right)^{-1}\left(\frac{\epsilon_{e}}{10^{-1}}\right)^{2}\left(\frac{\epsilon_{B}}{10^{-3}}\right)^{1 / 2} n^{1 / 2}\left(\frac{g_{m}}{0.087}\right) \\
& \times\left(\frac{\gamma_{0}}{10^{2.5}}\right)^{2} \mathrm{~Hz}, \\
\nu_{c, r}\left(t_{\times}\right)= & 2.8 \times 10^{18}\left(\frac{1+z}{2}\right)^{-1}\left(\frac{E_{\text {iso }}}{10^{53} \mathrm{ergs}}\right)^{-2 / 3}\left(\frac{\epsilon_{B}}{10^{-3}}\right)^{-3 / 2} n^{-5 / 6} \\
\times & \left(\frac{g_{c}}{0.128}\right)\left(\frac{\gamma_{0}}{10^{2.5}}\right)^{4 / 3} \mathrm{~Hz}, \\
F_{\nu, \text { max }, r}\left(t_{\times}\right)= & 10\left(\frac{1+z}{2}\right)\left(\frac{\epsilon_{B}}{10^{-3}}\right)^{1 / 2} n^{1 / 2}\left(\frac{E_{\text {iso }}}{10^{53} \mathrm{ergs}}\right)\left(\frac{D_{L}}{10^{28} \mathrm{~cm}}\right)^{-2} \\
& \times\left(\frac{g_{\max }}{2.44}\right)\left(\frac{\gamma_{0}}{10^{2.5}}\right) \mathrm{Jy},
\end{aligned}
$$

for the thin shell (i.e. Newtonian) case, and

$$
\begin{aligned}
\nu_{m, r}(T)= & 5.1 \times 10^{11}\left(\frac{1+z}{2}\right)^{-1}\left(\frac{\epsilon_{e}}{10^{-1}}\right)^{2}\left(\frac{\epsilon_{B}}{10^{-3}}\right)^{1 / 2} n^{1 / 2}\left(\frac{g_{m}}{0.087}\right)\left(\frac{\gamma_{0}}{10^{2.5}}\right)^{2} \mathrm{~Hz}, \\
\nu_{c, r}(T)= & 1.7 \times 10^{18}\left(\frac{1+z}{2}\right)^{-1 / 2}\left(\frac{\epsilon_{B}}{10^{-3}}\right)^{-3 / 2} n^{-1}\left(\frac{E_{\text {iso }}}{10^{53} \mathrm{ergs}}\right)^{-1 / 2}\left(\frac{g_{c}}{0.128}\right) \\
& \times\left(\frac{T}{10^{2} \mathrm{~s}}\right)^{-1 / 2} \mathrm{~Hz}
\end{aligned}
$$




$$
\begin{aligned}
F_{\nu, \text { max }, r}(T)= & 2.3\left(\frac{1+z}{2}\right)^{7 / 4}\left(\frac{E_{\text {iso }}}{10^{53} \mathrm{ergs}}\right)^{5 / 4}\left(\frac{\epsilon_{B}}{10^{-3}}\right)^{1 / 2} n^{1 / 4}\left(\frac{g_{\max }}{2.44}\right)\left(\frac{D_{L}}{10^{28} \mathrm{~cm}}\right)^{-2} \\
& \times\left(\frac{T}{10^{2} \mathrm{~s}}\right)^{-3 / 4}\left(\frac{\gamma_{0}}{10^{2.5}}\right)^{-1} \mathrm{Jy},
\end{aligned}
$$

for the thick shell (i.e. relativistic) case. Here, the GRB duration $\mathrm{T}$ is given by the shell width $\Delta_{0} / c$, according to the internal shock model. As derived by Sari \& Piran (1999a) and Kobayashi (2000), the typical synchrotron frequency and peak flux evolve as $\nu_{m, r} \propto t^{6}$, $F_{\nu, \max , r} \propto t^{3 / 2}$ at $t<t_{\times}$, and $\nu_{m, r} \propto t^{-54 / 35}, F_{\nu, \max , r} \propto t^{-34 / 35}$ at $t>t_{\times}$, for the thin-shell case; $\nu_{m, r}=$ constant, $F_{\nu, \max , r} \propto t^{1 / 2}$ at $t<T$, and $\nu_{m, r} \propto t^{-73 / 48}, F_{\nu, \max , r} \propto t^{-47 / 48}$ at $t>T$, for the thick-shell case. Before the crossing time, the cooling frequency evolve as $\nu_{c, r} \propto t^{-2}$ for the thin-shell case and $\nu_{c, r} \propto t^{-1}$ for the thick-shell case; after the crossing time, $\nu_{c, r}$ turns into the cutoff frequency due to synchrotron cooling of electrons without acceleration, and it evolves the same as $\nu_{m, r}$ for both cases. According to the emission properties shown above, we derive light curves of the emission from the forward- and reverse-shocked regions and thus can constrain the model parameters by fitting the early afterglow of GRB 050525A.

\section{Constraints on Parameters of GRB 050525A}

From the emission features described by equations (1), (2), (5), (6), (8), and (9), we see that the optical band is commonly between the typical frequency $\nu_{m}$ and cooling frequency $\nu_{c}$ in both forward and reverse shocks around the crossing time. Thus, the optical light curve of GRB 050525A can be fitted by the superposition of two broken power-laws: (1) forward shock: $F_{\nu, f} \propto t_{d}^{1 / 2}$ for $t_{d}<t_{m, f}$, and $F_{\nu, f} \propto t_{d}^{3(1-p) / 4}$ for $t_{d}>t_{m, f}$; (2) reverse shock: $F_{\nu, r} \propto t_{d}^{3(2 p-1) / 2}$ for $t_{d}<t_{\times}$, and $F_{\nu, r} \propto t_{d}^{(-27 p+7) / 35}$ for $t_{d}>t_{\times}$. These scaling laws are valid for the thin-shell case, in which the reverse shock decelerates the shell insignificantly (Kobayashi 2000).

In Figure 1 we present the R-band afterglow light curve of GRB 050525A between May $25.0062 \mathrm{UT}(\Delta t \approx 0.004$ days $)$ and May $25.5333 \mathrm{UT}(\Delta t \approx 0.531$ days $)$. We find that, $t_{m, f} \approx 0.027$ days, $F_{\nu, \max , f} \approx 0.719 \mathrm{mJy}$, and $p \approx 2.3$ can fit the light curve well for the component of forward shock emission. Given redshift $z=0.606$ (Foley et al. 2005), the corresponding luminosity distance is $D_{L}=1.1 \times 10^{28} \mathrm{~cm}$. Using equations (3) and (4), we obtain

$$
\epsilon_{e} \simeq 8.3 n^{1 / 4}\left(\frac{E_{\text {iso }}}{10^{53} \mathrm{ergs}}\right)^{1 / 4}
$$

and

$$
\epsilon_{B} \simeq 1.0 \times 10^{-5} n^{-1}\left(\frac{E_{\text {iso }}}{10^{53} \mathrm{ergs}}\right)^{-2} .
$$


Furthermore, from the flux inferred by the light curve of the reverse-shock emission (depicted as a dashed line in Figure 1, where we see a flux $F_{\nu, r}=0.75$ mJy at $t=0.01$ days), using equations (5) and (7), we further obtain

$$
\left(\frac{E_{\text {iso }}}{10^{53} \mathrm{ergs}}\right)^{1.658}\left(\frac{\epsilon_{B}}{10^{-3}}\right)^{0.825}\left(\frac{\epsilon_{e}}{10^{-1}}\right)^{1.3} n^{0.167}\left(\frac{\gamma_{0}}{10^{2.5}}\right)^{-2.965} \simeq 5.9
$$

By simple algebra from equations (11), (12) and (13), we obtain a constraint on the initial Lorentz factor of GRB ejecta,

$$
\gamma_{0}=121\left(\frac{E_{\text {iso }}}{10^{53} \mathrm{ergs}}\right)^{0.112}\left(\frac{n}{1 \mathrm{~cm}^{-3}}\right)^{-0.112},
$$

which is weakly dependent on the isotropic-equivalent kinetic energy $E_{i s o}$ and interstellar medium density $n$. Assuming the efficiency of energy conversion is $\eta=0.5, E_{i s o}$ is approximately equal to the detected isotropic-equivalent $\gamma$-ray energy, i.e. $1.2 \times 10^{53} \mathrm{ergs}$. Thus, we can obtain a mutual constraint between $\epsilon_{B}$ and $\epsilon_{e}$ from equations (11) and (12). By eliminating the medium density $n$, we have $\epsilon_{B}=4 \times 10^{-6} \epsilon_{e}^{-4}$. Moreover, as a natural consequence of $\epsilon_{e}<1$, we have $\gamma_{0}>116, \epsilon_{B}>4 \times 10^{-6}$, and $n<2 \mathrm{~cm}^{-3}$. These constraints are more clear than the cases of GRB 990123 (Sari \& Piran 1999b), GRB 021004 (Kobayashi \& Zhang 2003a) and GRB 021211 (Wei 2003). Our preliminary fitting to the afterglow observations of GRB $050525 \mathrm{~A}$ update to May $25.5333 \mathrm{UT}$ ( $\Delta t \approx 0.531$ days) is shown in Figure 1.

Additionally, we also give a constraint on the parameters for the thick-shell case. We can find from a fitting similar to that shown above that, the crossing time of the reverse shock, i.e., the GRB duration $T$ is approximated by

$$
T \simeq 553\left(\frac{E_{i s o}}{10^{53} \mathrm{ergs}}\right)^{0.06}\left(\frac{n}{1 \mathrm{~cm}^{-3}}\right)^{-0.06}\left(\frac{\gamma_{0}}{10^{2.5}}\right)^{0.25} \mathrm{~s},
$$

which is inconsistent with the observed $T_{90} \approx 10 \mathrm{~s}$ (Markwardt et al. 2005; Gotz et al. 2005). This implies that GRB 050525A seems to be a thin-shell case.

\section{Discussion and Conclusions}

The optical afterglow of GRB $050525 \mathrm{~A}$ rebrightened starting at $\sim 0.01$ days and ending at $\sim 0.03$ days, which was followed by an initial power-law decay. In this paper, we have shown that this early emission feature can be interpreted as the reverse shock emission superposed by the forward shock emission in an interstellar medium environment. A good fitting to the observed light curve is shown in Figure 1. We further find the initial Lorentz 
factor of the ejecta $\gamma_{0}>120$. Some other model parameters are also constrained: the magnetic energy fraction $\epsilon_{B}>4 \times 10^{-6}$ and the medium density $n<2 \mathrm{~cm}^{-3}$. These limits are consistent with those from the other very-early optical afterglows observed so far (Sari \& Piran 1999b; Kobayashi \& Zhang 2003a; Wei 2003; Panaitescu \& Kumar 2004b; Nakar \& Piran 2005) and with the values of the forward-shock parameters (Panaitescu \& Kumar 2001).

Our model is simplified under some assumptions: First, the reverse- and forward-shocked regions have the same electron and magnetic energy fractions, requiring that the ejecta is not initially magnetized. This requirement seems to be relaxed for GRB 990123 (Fan et al. 2002; Zhang, Kobayashi, \& Mészáros 2003). The interaction of a magnetized ejecta with its surrounding medium has been investigated in details (Fan, Wei, \& Wang 2004; Zhang \& Kobayashi 2005). Second, we neglect the effect of inverse Compton scattering (ICS) on the reverse shock emission in $\S 2$. As shown by Wang, Dai, \& Lu (2001a, b), this effect not only decreases the cooling frequency but also produces higher-energy emission. However, this decrease does not affect the limits derived above because the cooling frequency is much larger than the optical band at the times of our interest. Third, the surrounding environment is assumed to be a uniform interstellar medium, in which case two types of light curves are expected for the reverse-forward-shock emission combination: a rebrightening light curve (type I) and a flattening light curve (type II) (Zhang, Kobayashi, \& Mészáros 2003). Early afterglows in wind environments have been studied by Chevalier, \& Li (2000), Wu et al. (2003), Kobayashi \& Zhang (2003b), Panaitescu \& Kumar (2004b), and Zou et al. (2005). Generally, a flattening light curve in optical band is expected in the wind model (Kobayashi \& Zhang 2003b; Zou, Wu, \& Dai 2005), i.e., there is only type II light curve for the reverse-forward-shock emission combination. Since the early optical afterglow of GRB 050525A shows a rebrightening (type I) light curve, the reverse-forward shock model in a wind environment for the early afterglow of GRB 050525A is not favored.

The observed break at $t_{j} \simeq 0.4$ days in the optical afterglow light curve of GRB 050525A (Mirabal, Bonfield, \& Schawinski 2005) may be due to an ultrarelativistic jet. This conclusion seems to be strengthened by Lulin Observatory around 17.7 hours after the burst (at which the R-band magnitude is about $21.3 \pm 0.1$, Chiang et al. 2005). If so, the jet's half opening angle $\theta=0.051\left(t_{j} / 0.4 \mathrm{~d}\right)^{3 / 8}\left(E_{i s o} / 10^{53} \mathrm{ergs}\right)^{-1 / 8}\left(n / 0.1 \mathrm{~cm}^{-3}\right)^{1 / 8}\left(\eta_{\gamma} / 0.5\right)^{1 / 8}$ (Sari, Piran, \& Halpern 1999) and thus the beaming-corrected gamma-ray energy $E_{j e t}=$ $1.3 \times 10^{50}\left(t_{j} / 0.4 \mathrm{~d}\right)^{3 / 4}\left(E_{\text {iso }} / 10^{53} \mathrm{ergs}\right)^{3 / 4}\left(n / 0.1 \mathrm{~cm}^{-3}\right)^{1 / 4}\left(\eta_{\gamma} / 0.5\right)^{1 / 4} \mathrm{ergs}$, which is by a factor of a few smaller than the mean energy release found by Frail et al. (2001) for $n \sim 0.1 \mathrm{~cm}^{-3}$ and $\eta_{\gamma} \sim 0.5$. Even so, this energy still satisfies the relation of Ghirlanda et al. (2004), $\left(E_{\text {jet }} / 10^{50} \mathrm{ergs}\right)=(1.12 \pm 0.12)\left[(1+z) E_{p} / 100 \mathrm{keV}\right]^{1.50 \pm 0.08}$ (see Dai, Liang, \& Xu 2004). 
Finally, what we want to point out is that besides the reverse-shock model discussed in this paper, the other plausible explanations of a rebrightening light curve include the density-jump medium (Dai \& Lu 2002; Lazzati et al. 2002), pure Poynting-flux injection (Dai \& Lu 1998), baryon-dominated injection (Rees \& Mészáros 1998; Granot, Nakar \& Piran 2003; Nakar, Piran \& Granot 2003; Björnsson et al. 2004), two-component jet (Berger et al. 2003; Huang et al. 2003), neutron-fed fireball (Beloborodov 2003), temporal fluctuation of $\epsilon_{e}$ and/or $\epsilon_{B}$ and departure of the electron distribution from a power law (Panaitescu \& Kumar 2004). Multiwavelength observations in the Swift era are expected to distinguish among these possibilities.

We thank Y. Z. Fan and L. J. Gou for constructive comments, X. F. Wu and Y. C. Zou for helpful discussions, and Y. F. Huang and X. Y. Wang for a careful reading of our manuscript. This work was supported by the National Natural Science Foundation of China (grants 10233010 and 10221001) and the Ministry of Science and Technology of China (NKBRSF G19990754).

\section{REFERENCES}

Akerlof, C., et al. 1999, Nature, 398,400

Band, D., et al. 2005, GCN 3466

Beloborodov, A. M. 2003, ApJ, 585, L19

Berger, E., et al. 2003, Nature, 426, 154

Björnsson, G., Gudmundusson, E. H., \& Johanesson, G. 2004, ApJ, 615, L77

Blake, C. H., et al. 2005, Nature, 435, 181

Chevalier, R. A., \& Li, Z. Y. 2000, ApJ, 536, 195

Chiang, P. S., Huang, K. Y., Ip, W. H., Urata, Y., Qiu, Y., \& Lou, Y. Q. 2005, GCN 3486

Cobb, B. E., \& Bailyn, C. D. 2005, GCN 3506

Cummings, J., et al. 2005, GCN 3479

Dai, Z. G., \& Cheng, K. S. 2001, ApJ, 558, L109

Dai, Z. G., \& Lu, T. 1998, Phys. Rev. Lett., 81, 4301 
Dai, Z. G., \& Lu, T. 2002, ApJ, 565, L87

Dai, Z. G., Liang, E. W., \& Xu, D. 2004, ApJ, 612, L101

Fan, Y. Z., Dai, Z. G., Huang, Y. F., \& Lu, T. 2002, Chinese J. Astron. Astrophys., 2, 449

Fan, Y. Z., Wei, D. M., \& Wang, C. F. 2004, A\&A, 424, 477

Fan, Y. Z., \& Wei, D. M. 2005, astro-ph/0506155

Foley, R. J., Chen, H. W., Bloom, J., \& Prochaska, J. X. 2005, GCN 3483

Fox, D. W., et al. 2003a, Nature, 422, 284

Fox, D. W., et al. 2003b, ApJ, 586, L5

Frail, D. A., et al. 2001, ApJ, 562, L55

Galama, T. J., et al. 1999, Nature, 398, 394

Ghirlanda, G., Ghisellini, G., \& Lazzati, D. 2004, ApJ, 616, 331

Golenetskii, S., et al. 2005, GCN 3474

Gotz, D., et al. 2005, GCN 3472

Granot, J., \& Sari, R. 2002, ApJ, 568, 820

Granot, J., Nakar, E. \& Piran, T. 2003, Nature, 426, 138

Homewood, A., et al. 2005, GCN 3491

Huang, Y. F., Wu, X. F., Dai, Z. G., Ma, H. T., \& Lu, T. 2004, ApJ, 605, 300

Katz, J. I. 1994, ApJ, 422, 248

Klotz, A., Boer, M., \& Atteia, J. L. 2005, GCN 3473

Klotz, A., et al. 2005, A\&A, submitted (astro-ph/0506259)

Kobayashi, S. 2000, ApJ, 545, 807

Kobayashi, S., \& Zhang, B. 2003a, ApJ, 582, L75

Kobayashi, S., \& Zhang, B. 2003b, ApJ, 597, 455

Lazzati, D., Rossi, E., Covino, S., Ghisellini, G., \& Malesani, D. 2002, A\&A, 396, L5 
Li, W., et al. 2003, ApJ, 586, L9

Malesani, D., et al. 2005, GCN 3469

Markwardt, C., et al. 2005, GCN 3467

Mészáros, P., \& Rees, M. J. 1997, 476, 232

Mészáros, P., \& Rees, M. J. 1999, 306, L39

Mészáros, P. 2002, ARA\&A, 40, 137

Milne, P. A., Williams, G. G., \& Park, H. S. 2005, GCN 3485

Mirabal, N., Bonfield, D., \& Schawinski, K. 2005, GCN 3488

Nakar, E., Piran, T., \& Granot, J. 2003, New A, 8, 495

Nakar, E., \& Piran, T. 2005, ApJ, 619, L147

Panaitescu, A., \& Kumar, P. 2001, ApJ, 560, L49

Panaitescu, A., \& Kumar, P. 2004a, MNRAS, 350, 213

Panaitescu, A., \& Kumar, P. 2004b, MNRAS, 353, 511

Piran, T. 2005, Rev. Mod. Phys., 76, 1143

Rees, M., \& Mészáros, P. 1998, ApJ, 496, 1

Rykoff, E. S., Yost, S. A., \& Swan, H. 2005, GCN 3465

Rykoff, E. S., Yost, S. A., Swan, H., \& Quimby, R. 2005, GCN 3468

Sari, R., \& Piran, T. 1995, ApJ, 455, L143

Sari, R., Piran, T., \& Narayan, R. 1998, ApJ, 497, L17

Sari, R., \& Piran, T. 1999a, ApJ, 520, 641

Sari, R., \& Piran, T. 1999b, ApJ, 517, L109

Sari, R., Piran, T., \& Halpern , J. P. 1999, ApJ, 519, L17

Torii, K., \& BenDaniel, M. 2005, GCN 3470

Vestrand, W. T. et al. 2005, Nature, 435, 178 
Wang, X. Y., Dai, Z. G., \& Lu, T. 2001a, ApJ, 546, L33

Wang, X. Y., Dai, Z. G., \& Lu, T. 2001b, ApJ, 556, 1010

Wei, D. M. 2003, A\&A, 402, L9

Wu, X. F., Dai, Z. G., Huang, Y. F., \& Lu, T. 2003, MNRAS, 342, 1131

Yanagisawa, K., Toda, H., \& Kawai, N. 2005, GCN 3489

Zhang, B., Kobayashi, S., \& Mészáros, P. 2003, ApJ, 595, 950

Zhang, B., \& Mészáros, P. 2004, Int. J. Mod. Phys. A, 19, 2385

Zhang, B, \& Kobayashi, S. 2005, ApJ, in press (astro-ph/0404140)

Zou, Y. C., Wu, X. F., \& Dai, Z. G. 2005, MNRAS, submitted 


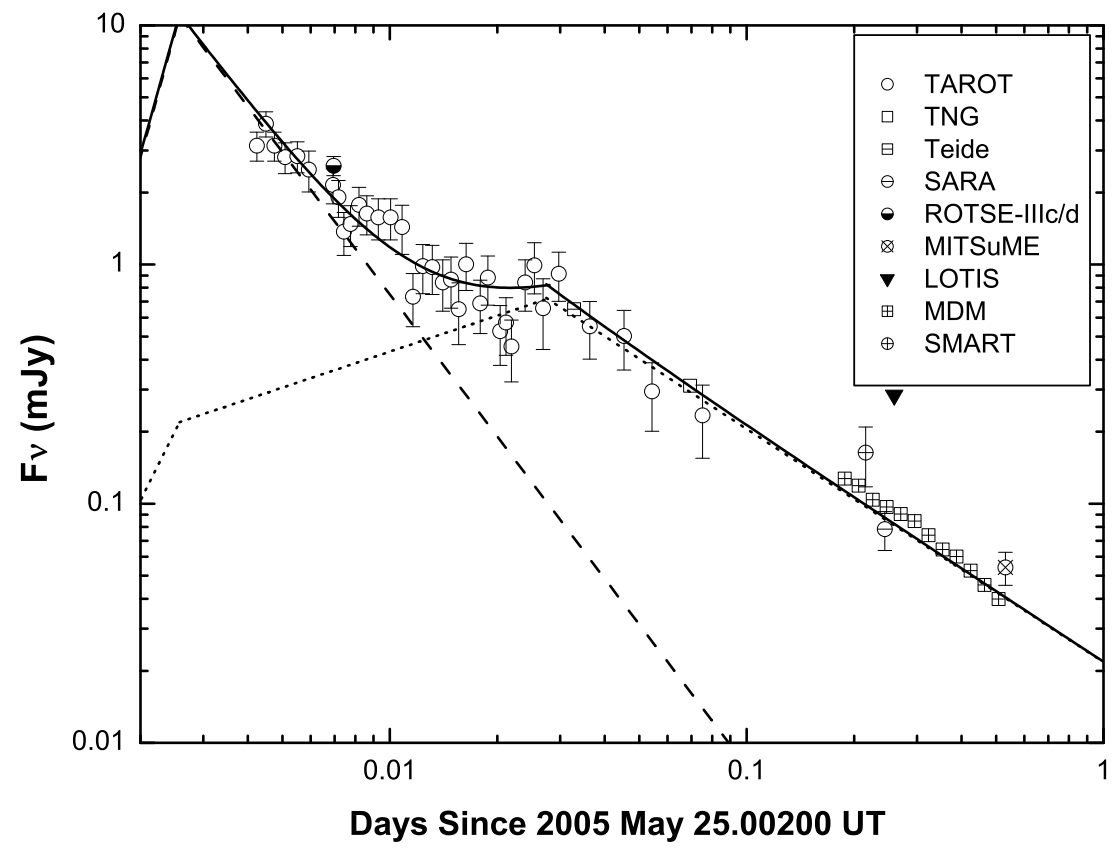

Fig. 1.- R-band afterglow light curve of GRB050525A. The fitting curve (solid line) is generated by superposition of forward shock emission (dotted line) and reverse shock emission (dashed line) with $\epsilon_{B}=0.0025, \epsilon_{e}=0.2, n=0.003 \mathrm{~cm}^{-3}, E_{\text {iso }}=10^{53} \mathrm{ergs}, p=2.3$, $\gamma_{0}=200$. These values are basically consistent with the thin-shell case. Data are taken from the GRB Coordinates Network (GCN): Malesani et al. (2005); Klotz, Boer, \& Atteia (2005); Torii \& BenDaniel (2005); Homewood et al. (2005); Rykoff et al. (2005); Yanagisawa, Toda \& Kawai (2005); Milne, Williams, \& Park (2005); Mirabal, Bonfield, \& Schawinski (2005); Cobb \& Bailyn (2005); Klotz et al. (2005). 